\title{
Synergies between SKA and ALMA: observations of Nearby Galaxies
}

\author{
Rosita Paladino ${ }^{* 1,2}$, Jan Brand ${ }^{2}$, Emanuela Orrú ${ }^{3}$, Viviana Casasola ${ }^{2}$, Elisabetta \\ Liuzzo $^{2}$, Marcella Massardi ${ }^{2}$, Arturo Mignano $^{2}$ \\ ${ }^{1}$ Università di Bologna; ${ }^{2}$ INAF-Istituto di Radioastronomia - Italian ALMA regional center; \\ ${ }^{3}$ ASTRON \\ E-mail: paladinodira.inaf.it
}

The past decade has seen amazing advances in radioastronomy, which led to the construction of brand-new instruments such as LOFAR and ALMA, and the updating of existing ones, e. g. JVLA and e-MERLIN. The SKA will be the spearhead of a future technological development and it will change the way astrophysical topics have been studied so far by opening up new frequency windows with unprecedented spatial resolution and sensitivity. The SKA location in the southern hemisphere makes it particularly suitable to complement ALMA, which is already giving exciting results both on the local and the more distant Universe.

Among the possible synergies between SKA and ALMA, we focus on the observations of nearby star forming galaxies. Star formation processes in galaxies involve all the components of the interstellar medium, so the only way to have a complete picture of them is through multifrequency observations.

ALMA observes gas and dust emission, while the SKA will trace both the free-free thermal and the non-thermal synchrotron emission. The spatial comparison between these components gives information about the contribution to star formation processes provided by magnetic fields and cosmic rays. The high spatial resolution achievable with ALMA and SKA will make it possible to compare these emissions on very small spatial scales, by resolving single molecular clouds in nearby galaxies.

By the time the SKA will start observing, ALMA will have already imaged many nearby galaxies in the southern hemisphere, for which no low frequency data at comparably high spatial resolution will be available. The SKA will fill this gap, and have a profound impact on the studies of nearby galaxies, making valuable contributions to our understanding of star formation processes, and of the role of magnetic fields and cosmic rays in them.

Advancing Astrophysics with the Square Kilometre Array

June 8-13, 2014

Giardini Naxos, Italy

\footnotetext{
* Speaker.
} 


\section{Introduction}

Star formation (SF) is a key astrophysical process, being the principal agent of galaxy formation and evolution. It is however difficult to formulate general theories about it, due to the wide range of physical processes involved. In the past decades, observational information has been obtained expanding the range of physical scales explored from individual protostellar and protoplanetary systems to molecular clouds and star clusters, to entire galaxies and systems of galaxies, see Krumholz (2014) for a comprehensive review on the current status of the field. Detailed observations of individual star-forming regions have been possible so far only in the Milky Way, while the extragalactic studies have been focused on characterizing the collective effects of star-formation processes on galactic and cosmological scales (see Kennicutt \& Evans, 2012, and references therein).

It is now clear that the large-scale star formation rate (SFR) is determined both by physical processes on galactic or extragalactic scales (such as the accretion of gas onto disks from satellite objects and intergalactic medium), and by processes operating on local cloud scales (from the cooling of the gas on kpc scales, to the formation of molecular clouds on pc scales, and the subsequent fragmentation and accretion of this molecular gas to form denser structures), for a recent review see Dobbs et al. (2013). The key processes influencing SF lie in the boundary between these physical scales. At present, there are no models that include all the physical processes necessary to simulate the transformation of the ISM from the galactic to the stellar scale, and future theories will certainly benefit from input from comparison with observations.

Nearby galaxies provide the opportunity to study galaxy processes on large scales, while still being close enough to reveal the local details, if high resolution and sensitivity are achieved. The advent of new observational facilities providing so far unattainable resolutions will make nearby galaxies key targets to address important questions, such as what is the importance of local (disk or cloud instability) and global effects (spiral density waves, tidal forces) in triggering SF, which is the role of magnetic fields, or how do environmental conditions or galaxy properties influence SF.

\section{Multi frequency studies}

Star formation processes take place in the cold gaseous medium of galaxies, but they involve in a very complex way all the components of the interstellar medium (ISM).

\section{- Gas}

The cold gas component can be observed in its atomic phase through the hyper-fine transition of hydrogen, occurring in the rest frame at $21 \mathrm{~cm}$, and in the molecular phase, mostly observable through molecular transitions in the millimetric domain. The most commonly used tracer of the molecular gas so far has been the carbon monoxide (CO): despite many drawbacks, its lines are the easiest to observe (see Bolatto et al. 2013 for a review). Essentially, $\mathrm{CO}$ emission traces molecular gas only over a limited dynamic range: since a minimum density is required to excite it, it does not trace all the molecular gas, especially if the metallicity is low; on the other end, $\mathrm{CO}$ becomes optically thick at very low total column densities. The use of $\mathrm{CO}$ isotopologues provides lower optical depths at the cost of weaker lines. Higher-J 
$\mathrm{CO}$ transitions and other molecular lines, such as $\mathrm{HCN}$ or $\mathrm{CS}$, are more suitable to trace warmer and denser gas.

\section{- Dust}

Despite containing only $1 \%$ of the total mass content in the ISM, dust plays an important role in SF since it helps in the gravitational collapse of molecular clouds, by acting as a cooling agent. In return, it is severely affected by the proximity of star formation, modifying the chemical composition of the circumstellar environs. From the observational point of view: dust absorbs radiation at higher frequencies where it can be optically thick, and reprocesses the energy, emitting at longer wavelengths, from IR to millimeter. Transformational results have been obtained with the Spitzer Space Telescope (Werner et al. 2004) and the Herschel Space Observatory (Pilbratt et al. 2010). At $\sim 1 \mathrm{~mm}$ the emission is close to the RayleighJeans limit, so the mm continuum emission provides a good tracer of dust column density and mass.

\section{- Cosmic rays and magnetic field}

In addition to the thermal gas and dust components, the ISM is permeated by a background of highly energetic particles, the cosmic ray component, consisting mainly of relativistic electrons and ions, tied to the galaxy by magnetic field.

It has become increasingly clear that cosmic rays and magnetic fields play a significant role in the star formation process, even though this role is far from being understood.

The tight FIR/radio continuum (RC) correlation (e.g. Condon 1992, Yun et al. 2001) is generally interpreted as a manifestation of the link between cosmic ray electrons (CRe) and star formation. CRe, responsible for diffuse synchrotron emission, are thought to be produced in expanding supernova remnants (SNRs), which are generally associated with massive star formation. The study of the origins and propagation mechanisms of the CRe is directly linked to the understanding of SF processes.

The role of magnetic fields at almost all stages of SF remains highly controversial (see Crutcher et al., 2012), in large part as a result of the lack of observational constraints. It is however clear that processes opposing gravitational collapse of the molecular clouds have to take place to justify the low efficiency of molecular clouds in forming stars.

Empirical relations between observational tracers of these different components have been exploited to study star formation rates in external galaxies (see Kennicutt \& Evans 2012 for a recent review). Radio continuum emission, which is not affected by dust obscuration, is widely used as a tracer of star formation in galaxies both at low and high redshifts. It will be particularly useful in the redshift range between 1 and 3, where the galaxies appear to be mostly dusty starbursts (e.g., Bouwens et al. 2009; Murphy et al. 2011). In order to calibrate radio continuum emission as a star formation tracer, observations of star forming regions in a large range of conditions, in the nearby Universe, will provide essential constraints. Furthermore, studies of low metallicity systems, such as dwarf irregular galaxies, can be used to extrapolate star formation laws in the unenriched early Universe. 
Table 1: SKA1-SUR and SKA1-MID parameters

\begin{tabular}{lcccc}
\hline \hline & $\begin{array}{c}\text { Freq* } \\
\text { GHz }\end{array}$ & $\begin{array}{c}\text { Max res* } \\
\text { arcsec }\end{array}$ & $\begin{array}{c}\text { Cont rms* } \\
\mu \mathrm{Jy} \cdot \mathrm{hr}^{-1 / 2}\end{array}$ & $\begin{array}{c}\text { scale @ 10 Mpc } \\
\mathrm{pc}\end{array}$ \\
\hline SKA1-SUR PAF2 & $0.650-1.67$ & $1.9-0.8$ & 5.6 & $92-39$ \\
SKA1-MID Band 2 & $0.950-1.76$ & $0.32-0.17$ & 0.96 & $15.5-8.3$ \\
SKA1-MID Band 4 & $2.8-5.2$ & $0.11-0.06$ & 0.45 & $5.4-2.9$ \\
SKA1-MID Band 5 & $4.6-13.8$ & $0.07-0.02$ & 0.52 & $3.4-0.97$ \\
\hline \hline
\end{tabular}

*From tables 6 and 7 of the SKA1 system baseline design (Dewdney et al., 2013)

Table 2: Some interesting lines observable with ALMA and the range of resolutions achievable

\begin{tabular}{ccccc}
\hline \hline Line & $\begin{array}{c}\text { restfreq } \\
\text { GHz }\end{array}$ & $\begin{array}{c}\text { Band } \\
\text { res range } \\
\text { arcsec }\end{array}$ & $\begin{array}{c}\text { range of scales } \\
\text { @ } 10 \mathrm{Mpc} \text { (in pc) }\end{array}$ \\
\hline $\mathrm{CO}(1-0)$ & 115.27 & 3 & $13-0.1$ & $630-5$ \\
$\mathrm{CO}(2-1)$ & 230.58 & 6 & $11-0.02$ & $533-1$ \\
$\mathrm{HCN}(1-0)$ & 88.63 & 3 & $13-0.1$ & $630-5$ \\
$\mathrm{HCN}^{2}(3-2)$ & 265.88 & 6 & $11-0.02$ & $533-1$ \\
$\mathrm{HCO}^{+}(1-0)$ & 85.16 & 3 & $13-0.1$ & $630-5$ \\
$\mathrm{HCO}^{+}(3-2)$ & 267.55 & 6 & $11-0.02$ & $533-1$ \\
$\mathrm{DCO}^{+}(1-0)$ & 70.73 & 2 & $6-0.05$ & $290-2.4$ \\
$\mathrm{DCO}^{+}(2-1)$ & 141.46 & 4 & $3-0.03$ & $145-1.4$ \\
\hline \hline
\end{tabular}

\section{Synergies between SKA and ALMA}

\subsection{Radio continuum and molecular emissions correlation}

The radio emission from galaxies at centimetric wavelengths is a combination of thermal and non-thermal radiation: both arising from massive star formation. Thermal emission, due to bremstrahlung radiation from star forming regions, is dominant at short wavelengths $(<20 \mathrm{~cm})$. At 3.6 $\mathrm{cm}, 30 \%$ of emission is thermal and can be directly related to the ionizing photon rate arising from newly formed massive stars (Condon 1992). In the low frequency range (wavelengths larger than $\sim 20 \mathrm{~cm}$ ), the dominant component is the non-thermal synchrotron emission from CR electrons accelerated by supernovae and spiraling in galactic magnetic fields.

The high resolution and sensitivity provided by SKA allow the observation of both the free-free thermal component and the non-thermal synchrotron emission in nearby galaxies, and the separation of their contribution on local scales. It will be possible to study the details of gas processing and to get a clear and detailed picture of star formation on large (kpc) scales, by decomposing the galaxies into individual sources and disentangling the contribution from AGNs. Furthermore, the high sensitivity provided by SKA will make it possible to observe the relativistic phase (magnetic 
field and cosmic rays) in faint regions, so far invisible, helping in clarifying which role this phase plays in SF processes.

ALMA allows observations of the molecular emission with comparable resolution and sensitivity. A correlation between radio continuum and $\mathrm{CO}$ emission has been found to hold in nearby galaxies, both on global (Adler et al, 1991; Murgia et al. 2002) and local scales (e.g. Murgia et al. 2005; Paladino et al. 2006, 2008; Schinnerer et al. 2013), but its origin is still not understood. The combination of SKA and ALMA will allow the study of this correlation down to spatial scales of the order of few parsecs (last columns of tables 1 and 2 list the range of spatial scales covered by SKA1 and ALMA). Emission from molecules such as $\mathrm{HCN}, \mathrm{HCO}+\mathrm{CN}$, less abundant than $\mathrm{CO}$ but tracing much denser gas, will be observable in nearby galaxies with the unprecedented sensitivity offered by ALMA. In very nearby galaxies (closer than $10 \mathrm{Mpc}$ ), even the internal structures of giant molecular clouds (GMCs) can be studied, and, thanks to ALMA polarization capabilities, it will be also possible to determine magnitude and direction of magnetic field in GMCs. A recent study on M33 GMCs (Li \& Henning 2011) has proven the possibility to determine the magnetic field orientation from the polarization of molecular lines. The comparison between large-scale galactic magnetic field (obtained from synchrotron polarization observations with SKA), and the clouds' magnetic fields is essential to clarify the origin and distribution of magnetic fields in GMCs, and hence their role in star formation processes.

ALMA also provides continuum observations in the millimetric range. These observations, complementing RC, IR and molecular line observations, allow the spatially resolved study of the spectral energy distribution, from centimeter to sub-millimeter wavelengths, allowing the investigation of the interaction between SF and nuclear activity. Furthermore, the dust is a particularly relevant tracer of interstellar matter with low heavy elements abundance.

\subsection{Observations of giant molecular clouds in nearby galaxies}

SKA and ALMA, when fully operational, will allow the extension of studies currently done only on galactic $\mathrm{H}$ II regions to nearby spiral and faint irregular galaxies.

One of the still open questions about star formation processes is whether they care about the galactic structure in which they happen. Star formation can be thought of as a localized process: the coldest gas in GMCs, at least three orders of magnitude smaller in size than a galaxy, collapses to form stars in cores, another order of magnitude smaller; however, large-scale dynamics may drive the evolution of GMCs (Koda et al. 2009).

Some of the properties of GMCs have been observed to be similar in different galaxies (Blitz et al. 2007; Bolatto et al. 2008; Donovan Meyer et al., 2013), suggesting that GMCs are unaware of the global environment. Evidence to the contrary has also been gathered during the past years: systematic variations in the Kennicutt-Schmidt relation (Schmidt 1959, Kennicutt 1998) have been found and interpreted as influence on star formation activity of global structural variations, such as galaxy type (Daddi et al. 2010; Leroy et al. 2013), condition in the galactic central region (Oka et al. 2001) and the morphology (Sheth et al. 2002; Momose et al. 2010).

On the theoretical side, various studies have found a dependence of GMC properties on galactic structures (Dobbs \& Bonnell 2006; Koda et al. 2009; Fujimoto et al. 2014). High spatial resolution radio observations of nearby galaxies with VLA or ATCA have allowed the study of compact sources such as HII regions and SNRs in starburst galaxies at distances up to $5 \mathrm{Mpc}$ (e. g. 
Table 3: Scaled flux of Cas A and W49A at 1.4 and $5 \mathrm{GHz}$, at various distances

\begin{tabular}{c|ccc|ccc}
\hline \hline Distance & \multicolumn{3}{|c}{ Cas A $(\mu \mathrm{Jy})$} & \multicolumn{3}{c}{ W49A $(\mu \mathrm{Jy})$} \\
\hline $\mathrm{Mpc}$ & 1.4 & 5 & $8 \mathrm{GHz}$ & 1.4 & 5 & $8 \mathrm{GHz}$ \\
\hline 10 & 177 & 58 & 48 & 93 & 114 & 131 \\
25 & 28 & 9 & 8 & 15 & 18 & 21 \\
50 & 7 & 2.3 & 2 & 3.7 & 4.6 & 5 \\
75 & 3 & 1 & 0.8 & 1.7 & 2 & 2.3 \\
100 & 2 & 0.6 & 0.5 & 0.9 & 1.1 & 1.3 \\
\hline \hline
\end{tabular}

Lacey et al. 1997; Gordon et al. 1999). To fully understand massive star formation and supernova evolution though, different types of galaxies with different properties must be surveyed in order to understand the effects of gas density and metallicity on star formation.

If star formation is really influenced by environmental effects, there must be a variation of GMCs properties. Obviously, GMCs in the Milky Way have been studied to a high level of precision (Larson 1981; Garcia et al. 2014), but observations in our own Galaxy are often hampered by our location inside its disk. Current observing facilities allow the study of GMCs in only a few galaxies (e.g. Hughes et al 2013) close enough to get the necessary resolution, while current limits in sensitivity prevent the observations of $\mathrm{CO}$-faint dwarf galaxies.

Aversa et al. (2011) recently studied a sample of star forming galaxies within $10 \mathrm{Mpc}$, to identify candidate star-forming regions in them. Due to current sensitivity limits only a few thermal sources have been detected. Higher sensitivity and spatial resolution will allow this kind of studies in more distant galaxies.

\section{SKA foreseen contribution to the field}

To estimate the capabilities of the SKA in detecting thermal and non-thermal compact sources in external galaxies, we use two prototypical sources in the Milky Way as benchmarks for analogous ones in external galaxies. The core-collapse SNR Cas A, with the highest luminosity and youngest age in the Galaxy (Baars et al. 1977; Fesen et al. 2006) will be our model for non-thermal sources, while W49A, the most luminous starforming region in the Milky Way (e.g. Galvan-Madrid 2013), will be our standard thermal source.

Cas A, at a distance of $2.8 \mathrm{kpc}$, has a flux density of 2260, 740 and $612 \mathrm{Jy}$, at $1.4,5$ and 8 $\mathrm{GHz}$, respectively (Baars et al, 1977), and W49A (at $14.1 \mathrm{kpc}$ ) has a flux density of 47.2, 57.5, 66 Jy at 1.4, 5 and $8 \mathrm{GHz}$, respectively (Mezger, Schraml \& Terzian 1967). We scaled these fluxes at distances up to $100 \mathrm{Mpc}$, results are reported in Table 3.

SKA1-MID - The SKA1-MID is particularly suitable for this kind of studies. Assuming that the available bands will be Band $2(0.950-1.76 \mathrm{GHz})$, Band 4 (2.8-5.2 GHz), and Band 5 (4.6-13.8 $\mathrm{GHz}$ ), the continuum sensitivity achievable (reported in Table 1) is enough to ensure $4 \sigma$ detections of thermal sources up to $75 \mathrm{Mpc}$ in $\sim 2 \mathrm{hrs}$ at $1.4 \mathrm{GHz}$, and $\sim 3 \mathrm{hrs}$ at $5 \mathrm{GHz} .4 \sigma$ detections of non-thermal sources will be possible in $\sim 5 \mathrm{hrs}$ at $1.4 \mathrm{GHz}$, and $<1 \mathrm{hr}$ at $5 \mathrm{GHz}$. 
In terms of spatial resolution, SKA1-MID will allow spatial resolution of the order of $\sim 50 \mathrm{pc}$ in $50 \mathrm{Mpc}$ distant galaxies at $1.4 \mathrm{GHz}$. The spatial scales will be smaller at higher frequencies. These resolutions are high enough to identify compact sources with sizes typical of the galactic GMCs $\sim 40$ pc (ranging from 20 to 100 pc; Solomon et al., 1987).

In SKA "early science" phase, with sensitivity about $50 \%$ of the full SKA1 specified level, the observations of these kinds of objects will be already feasible in a reasonable amount of time, compared to the current existing facilities. In less than four hours of observations it will be already possible to detect, with $\mathrm{S} / \mathrm{N}$ larger than 4 , thermal and non-thermal sources in galaxies at distances up to $50 \mathrm{Mpc}$.

SKA2 - Obviously, SKA2 sensitivity, 10 times higher than what is obtained in phase 1, and its resolution, 20 times better, will bring about a significant leap in the current way we study nearby galaxies. Thermal and non-thermal compact sources up to distances of $\sim 100 \mathrm{Mpc}$ will be detected, with a $4 \sigma \mathrm{S} / \mathrm{N}$, in few minutes. In galaxies at distances $<10 \mathrm{Mpc}$, SKA2 resolution will correspond to spatial scales of the order of a few tenths of a pc. Relatively face-on spirals such as M 33 present an excellent environment in which to examine the global aspects of massive star formation as traced through their HII region populations. The SKA2 resolution of $\sim 15$ milli-arcsec at $5 \mathrm{GHz}$ corresponds to nearly $0.05 \mathrm{pc}$ in $\mathrm{M} 33$. This is the scale of ultra-compact $\mathrm{H}$ II regions, which are not accessible to optical or even near-IR observations, since they are deeply embedded in their parent molecular cloud. In galaxies of the Local Group, the SKA2 will access scales explored so far only in our own Galaxy.

ALMA will observe with comparable resolution the millimetric counterpart of these sources. Scaling the millimetric flux of W49A (Galvan-Madrid et al. 2014) to larger distances, we verified the possibility to detect dust in typical starforming regions with continuum observations. Detection of lines will be possible for the more abundant species, such as $\mathrm{CO}$ and its isotopologues, up to $10 \mathrm{Mpc}$ distances. If the ratio between molecular species is the same as in our own Galaxy, a few relatively abundant molecules $\left(\mathrm{HCO}^{+}, \mathrm{DCO}^{+}\right.$, etc.) will also be detected, with low $\mathrm{S} / \mathrm{N}$. However, ALMA will provide information on the chemistry of faint regions and in low metallicity environments, which is still not well known. In particular, ALMA band 2, not yet available but possibly operational when SKA2 will start observing, will allow observations of deuterated molecules (some of them are listed in Table 2). These molecules drive the chemistry in highly embedded regions, where $\mathrm{CO}$ is depleted, in addition in cold, dark clouds, where some molecules are highly enriched in deuterium due to chemical fractionation. For these reasons, observations of them in external galaxies are extremely interesting but so far impossible due to sensitivity limits.

\section{REFERENCES}

Adler, D. S., Allen, R. J., \& Lo, K. Y. 1991, ApJ, 382, 475

Aversa, A. G., Johnson, K. E., Brogan, C. L. et al., 2011, AJ, 141, 125

Baars, J. W. M., Genzel, R., Pauliny-Toth, I. I. K., \& Witzel, A., 1977, A\&A, 61, 99

Blitz, L., Fukui, Y., Kawamura, A. et al., 2007, prpl.conf 81B

Bolatto, A. D., Leroy, A. K., Rosolowsky, E. et al., 2008, IAU Symposium, 255, 274

Bolatto, A. D., Wolfire, M., Leroy, A. K., 2013,ARA\&A, 51, 207B

Bouwens, R. J., Illingworth, G. D., Franx, M. et al., 2009, ApJ, 705, 936 
Condon, J. J., 1992, ARA\&A, 30, 575

Crutcher, R. M., 2012, ARA\&A, 50, 29

Daddi, E., Elbaz, D., Walter, F. et al., 2010, ApJ, 714, L118

Dewdney, P., Turner, W., Millenaar, R., McCool, R., Lazio, J., Cornwell, T., 2013, "SKA1 System Baseline Design", Document number SKA-TEL-SKO-DD-001 Revision 1

Dobbs, C. L., Bonnell, I. A., Pringle, J. E., 2006, MNRAS, 371,1663

Dobbs, C. L., Krumholz, M. R., Ballesteros-Paredes, J. et al., 2013, arXiv1312.3223

Donovan Meyer, J., Koda, J., Momose, R., et al., 2013, ApJ, 772, 107

Fesen, R. A., Hammell, M. C., Morse, J. et al., 2006, ApJ, 645, 283

Fujimoto, Y., Tasker, E. J., Wakayama, M. et al., 2014, MNRAS, 439, 936

Galván-Madrid, R., Liu, H. B., Zhang, Z.-Y. et al., 2013, ApJ, 779, 121

Galván-Madrid, R., Liu, H. B., Manara, C.-F. et al., 2014, ApJ, 570, L9

García, P., Bronfman, L., Nyman, L.- $\AA ., 2$ 2014, ApJS, 212, 2

Gordon, S. M., Duric, N., Kirshner, R. P., 1999, ApJS, 120, 247

Hughes, A., Meidt, S. E., Colombo, D. et al., 2013, ApJ, 779, 46

Kennicutt, R. C. 1998, ApJ, 498, 541

Kennicutt, R. C., Evans, N. J., 2012, ARA\&A, 50, 531

Koda, J., Scoville, N., Sawada, T., et al., 2009, ApJ, 700, L132

Krumholz, M. R., 2014, arXiv1402.0867

Lacey, C., Duric, N., Goss, W. M., 1997, ApJS, 109, 417

Larson, R. B., 1981, MNRAS, 194, 809

Leroy, A. K., Walter, F., Sandstrom, K. et al., 2013, AJ, 146, 19

Li, H.-B., Henning, T., 2011, Nature, 479, 499

Mezger, P. G., Schraml, J., Terzian, Y., 1967, AJ, 72, 817

Momose, R., Okumura, S. K., Koda, J. et al, 2010, ApJ, 721, 383

Murgia, M., Crapsi, A., Moscadelli, L., \& Gregorini, L., 2002, A\&A, 385, 412

Murgia, M., Helfer, T. T., Ekers, R., et al., 2005, A\&A, 437, 389

Murphy, E. J., Chary, R.-R., Dickinson, M.,et al., 2011, ApJ, 732, 126

Oka, T., Hasegawa, T., Sato, F., 2001, ApJ, 562, 348

Paladino, R., Murgia, M., Helfer, T. T., et al., 2006, A\&A, 456, 779

Paladino, R., Murgia, M., Tarchi, A., et al, 2008, A\&A, 485, 679

Pilbratt, G. L., Riedinger, J. R., Passvogel, T. et al., 2010, A\&A, 26A, 518L

Schmidt, M., 1959, ApJ, 129, 243

Schinnerer, E., Meidt, S. E., Pety, J., et al., 2013, ApJ, 779, 42

Sheth, K., Vogel, S. N., Regan, M. W. et al., 2002, AJ, 124, 2581

Solomon, P. M., Rivolo, A. R., Barrett, J. et al., 1987, ApJ, 319, 730

Werner, M. W, Roellig, T. L., Low, F. J. et al., 2004, ApJS, 154, 1

Yun, M. S., Reddy, N. A., \& Condon, J. J. 2001, ApJ, 554, 803 